\title{
SWCam: the short wavelength camera for the CCAT Observatory
}

\section{Gordon J. Stacey, Stephen Parshley, Thomas Nikola, German Cortes-Medellin, Justin Schoenwald, et al.}

Gordon J. Stacey, Stephen Parshley, Thomas Nikola, German CortesMedellin, Justin Schoenwald, Ganesh Rajagopalan, Michael D. Niemack, Tim Jenness, Patricio Gallardo, Brian Koopman, Charles D. Dowell, Peter K. Day, Matthew I. Hollister, Attila Kovacs, Henry G. LeDuc, Christopher M. McKenney, Ryan M. Monroe, Hiroshige Yoshida, Jonas Zmuidzinas, Loren J. Swenson, Simon J. Radford, Hien Trong Nguyen, Anthony K. Mroczkowski, Jason Glenn, Jordan Wheeler, Philip Maloney, Spencer Brugger, Joseph D. Adams, Frank Bertoldi, Reinhold Schaaf, Mark Halpern, Douglas Scott, Galen Marsden, Jack Sayers, Scott Chapman, Joaquin D. Vieira, "SWCam: the short wavelength camera for the CCAT Observatory," Proc. SPIE 9153, Millimeter, Submillimeter, and Far-Infrared Detectors and Instrumentation for Astronomy VII, 91530L (19 August 2014); doi: 10.1117/12.2057101

Event: SPIE Astronomical Telescopes + Instrumentation, 2014, Montréal, Quebec, Canada 


\title{
SWCam: the short wavelength camera for the CCAT observatory
}

\author{
Gordon J. Stacey ${ }^{*}$, Stephen Parshley ${ }^{\mathrm{a}}$, Thomas Nikola ${ }^{\mathrm{a}}$, German Cortes-Medellin ${ }^{\mathrm{a}}$, Justin \\ Schoenwald ${ }^{\mathrm{a}}$, Ganesan Rajagopalan ${ }^{\mathrm{a}}$, Michael D. Niemack ${ }^{\mathrm{a}}$, Tim Jenness ${ }^{\mathrm{a}}$, Patricio Gallardo ${ }^{\mathrm{a}}$, Brian \\ Koopman $^{\mathrm{a}}$, Charles D. Dowell ${ }^{\mathrm{b}}$, Peter K. Day ${ }^{\mathrm{c}}$, Matthew I. Hollister ${ }^{\mathrm{b}}$, Attila Kovacs, ${ }^{\mathrm{b}, \mathrm{d}}$, Henry G. \\ LeDuc $^{c}$, Christopher M. McKenney ${ }^{\mathrm{b}}$, Ryan M. Monroe ${ }^{\mathrm{c}}$, Hiroshige Yoshida ${ }^{\mathrm{b}}$, Jonas Zmuidzinas ${ }^{\mathrm{b}}$, \\ Loren J. Swenson ${ }^{\mathrm{b}}$, Simon J. Radford ${ }^{\mathrm{b}}$, Hien Trong Nguyen ${ }^{\mathrm{c}}$, Anthony K. Mroczkowski ${ }^{\mathrm{b}}$, Jason \\ Glenn $^{\mathrm{e}}$, Jordan Wheeler ${ }^{\mathrm{e}}$, Phil Maloney ${ }^{\mathrm{e}}$, Spencer Brugger ${ }^{\mathrm{e}}$, Joseph D. Adams ${ }^{\mathrm{f}}$, Frank Bertoldi ${ }^{\mathrm{g}}$, \\ Reinhold Schaaf ${ }^{\mathrm{g}}$, Mark Halpern ${ }^{\mathrm{h}}$, Douglas Scott ${ }^{\mathrm{h}}$, Gaelen Marsden ${ }^{\mathrm{h}}$, Jack Sayers ${ }^{\mathrm{b}}$, Scott Chapman ${ }^{\mathrm{i}}$, \\ Joaquin D. Vieira ${ }^{\mathrm{b}}$ \\ ${ }^{a}$ Cornell University, Ithaca, NY 14853, USA; \\ ${ }^{\mathrm{b}}$ California Institute of Technology USA \\ ${ }^{\mathrm{c}}$ Jet Propulsion Lab USA \\ ${ }^{\mathrm{d}}$ University of Minnesota USA \\ ${ }^{\mathrm{e}}$ University of Colorado, Boulder USA \\ fSOFIA-USRA, NASA/Armstrong Flight Research Center, Science and Aircraft Integration Facility, \\ Palmdale, CA 93550, USA;USRA \\ ${ }^{g}$ Rheinische Friedrich-Wilhelms-University, Bonn Germany \\ ${ }^{\mathrm{h}}$ The University of British Columbia Canada \\ ${ }^{\mathrm{i}}$ Dalhousie University Canada
}

\begin{abstract}
We describe the Short Wavelength Camera (SWCam) for the CCAT observatory including the primary science drivers, the coupling of the science drivers to the instrument requirements, the resulting implementation of the design, and its performance expectations at first light. CCAT is a $25 \mathrm{~m}$ submillimeter telescope planned to operate at 5600 meters, near the summit of Cerro Chajnantor in the Atacama Desert in northern Chile. CCAT is designed to give a total wave front error of $12.5 \mu \mathrm{m} \mathrm{rms,} \mathrm{so} \mathrm{that} \mathrm{combined} \mathrm{with} \mathrm{its} \mathrm{high} \mathrm{and} \mathrm{exceptionally} \mathrm{dry} \mathrm{site,} \mathrm{the} \mathrm{facility} \mathrm{will} \mathrm{provide} \mathrm{unsurpassed}$ point source sensitivity deep into the short submillimeter bands to wavelengths as short as the $200 \mu \mathrm{m}$ telluric window. The SWCam system consists of 7 sub-cameras that address 4 different telluric windows: 4 subcameras at $350 \mu \mathrm{m}, 1$ at $450 \mu \mathrm{m}, 1$ at $850 \mu \mathrm{m}$, and 1 at $2 \mathrm{~mm}$ wavelength. Each sub-camera has a 6' diameter field of view, so that the total instantaneous field of view for SWCam is equivalent to a 16' diameter circle. Each focal plane is populated with near unit filling factor arrays of Lumped Element Kinetic Inductance Detectors (LEKIDs) with pixels scaled to subtend an solid angle of $(\lambda / D)^{2}$ on the sky. The total pixel count is 57,160 . We expect background limited performance at each wavelength, and to be able to map $>35^{(\circ) 2}$ of sky to $5 \sigma$ on the confusion noise at each wavelength per year with this first light instrument. Our primary science goal is to resolve the Cosmic Far-IR Background (CIRB) in our four colors so that we may explore the star and galaxy formation history of the Universe extending to within 500 million years of the Big Bang. CCAT's large and high-accuracy aperture, its fast slewing speed, use of instruments with large format arrays, and being located at a superb site enables mapping speeds of up to three orders of magnitude larger than contemporary or near future facilities and makes it uniquely sensitive, especially in the short submm bands.
\end{abstract}

Keywords: CCAT telescope, submillimeter cameras, LEKID detectors, submillimeter galaxies, high redshift universe

"gjs12@cornell.edu; phone 1 (607) 255-5900; submm.astro.cornell.edu; ccatobservatory.org

Millimeter, Submillimeter, and Far-Infrared Detectors and Instrumentation for Astronomy VII, edited by Wayne S. Holland, Jonas Zmuidzinas, Proc. of SPIE Vol. 9153, 91530L

(C) 2014 SPIE · CCC code: 0277-786X/14/\$18 - doi: 10.1117/12.2057101

Proc. of SPIE Vol. 9153 91530L-1 


\section{INTRODUCTION}

CCAT is a $25 \mathrm{~m}$ submillimeter telescope planned to operate at 5600 meters, near the summit of Cerro Chajnantor in the Atacama Desert in northern Chile ${ }^{1}$. CCAT is designed to give a total wave front error of $12.5 \mu \mathrm{m} \mathrm{rms}$, so that combined with its high and exceptionally dry site, the facility will provide unsurpassed point source sensitivity deep into the short submillimeter bands to wavelengths as short as the $200 \mu \mathrm{m}$ telluric window. The shortest wavelength envisioned for routine operation is the $350 \mu \mathrm{m}$ window in which CCAT will routinely deliver 3.5" resolution images with a point source sensitivity (NEFD $\sim 13 \mathrm{mJy} \mathrm{s}^{1 / 2}$ ) better than that of the entire ALMA array. The large format arrays that will be fielded within CCAT instrumentation thereby ensure mapping speeds at wavelengths shorter than $1 \mathrm{~mm}$ that are ten to many thousands of times faster than any other facilities in existence or on the near term horizon. Here we present the preliminary design for the first light instrument on CCAT, the Short Wavelength Camera (SWCam). SWCam is designed to pursue the primary science of the CCAT facility, which is to measure the star formation in galaxies through cosmic time, through deep, wide-field imaging of the Cosmic Far-Infrared Background (CIRB) in the 350, 450, $850 \mu \mathrm{m}$ and $2.0 \mathrm{~mm}$ telluric widows. The fundamental parameters for SWCam (pixel size, numbers of pixels, and numbers of pixels at each color) are all driven by this science, but the capabilities of SWCam also fulfill the science requirements for other CCAT programs including studies of star formation within the Milky Way and nearby galaxies, and the astrophysics of galaxy clusters. The construction of SWCam is a collaborative effort amongst the CCAT member institutions, and several key personnel are shared amongst institutions. The enabling technology for SWCam is the KID arrays, which are also envisioned for the other two direct detection CCAT instruments (LWCam and X-Spec) that are also under development.

The SWCam/CCAT combination is uniquely powerful. No other current or foreseeable facility competes with SWCam/CCAT for deep, wide-field continuum imaging in the short-submm bands. The high-sensitivity, high-spatial resolution SWCam surveys will break through the confusion limit to resolve most of the cosmic far infrared background (CIRB), thereby tracing the star formation history of the Universe. Wide-field mosaics will trace the large-scale structure of star-forming galaxies through cosmic time and reveal "rare beasts" in the high-z Universe.

\section{SCIENTIFIC INSPIRATION}

Understanding the formation and evolution of galaxies is a major goal of $21^{\text {st }}$ century astrophysics, and is the main science driver for SWCam on CCAT. The presence of dust at very early times means that observations in the submm bands are key. Dust absorbs the stellar radiation in star-forming galaxies as well as the accretion radiation from buried Active Galactic Nuclei (AGN), making it problematic to observe these processes in the optical bands. The dust reradiates the absorbed energy into the rest frame far-IR bands, so that the dust continuum traces the stellar/AGN luminosity. The presence of dust is clearly important over the entire star formation history of the Universe - fully $50 \%$ of the energy ever released from nucleosynthesis or accretion has been reprocessed by dust to produce the CIRB and the re-radiated fraction is even higher at large redshifts than in the local Universe ${ }^{2}$.

We refer to all high redshift galaxies that emit an appreciable fraction of their luminosity in the far-IR bands, whether the ultimate source of this luminosity is star formation or AGN activity, as "submillimeter galaxies" or SMGs. High luminosity SMGs have been, and are being investigated in the FIR/submm/mm continuum by SCUBA, MAMBO, AzTEC, LABOCA, Bolocam, SHARC II, BLAST, and the Herschel Space Observatory, and tens of thousands have been discovered. Therefore, one might ask, why study SMGs with CCAT? These previous studies have been severely source confusion limited due to their limited angular resolution, making source counterpart identification difficult, and robust studies of the luminosity function nearly impossible - only about $10 \%$ of the CIRB is directly resolved by these studies. The confusion is primarily due to the very robust star formation activity that occurs at redshifts 1-3. Sources at these redshifts can dominate the CIRB, so that it is difficult to find the fainter emission from higher redshift sources. However, CCAT's $25 \mathrm{~m}$ high accuracy aperture at its unsurpassed site enables routine operations in the short-submm bands, where the 3.5 " beams of SWCam will break through the confusion barrier (Figure 1). However, to truly understand the star formation history of the Universe, we not only need to break the confusion, but we also need to survey large areas on the sky, so that we detect tens to hundreds of thousands of galaxies per redshift interval back to the earliest times. Within five years of first light, SWCam imaging will resolve $>80 \%$ of the CIRB over hundreds of square degrees, detecting millions of high z galaxies, thereby tracing the luminosity function of dusty star-forming galaxies over cosmic time. 


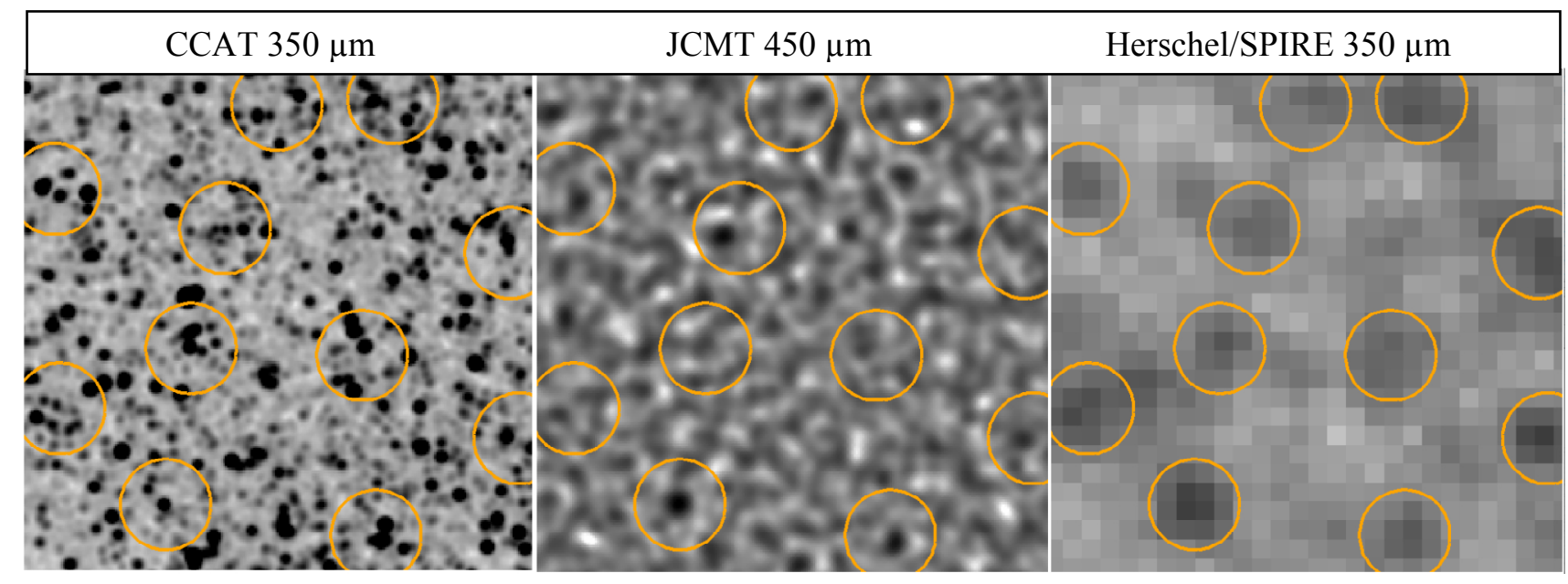

Figure 1: 4' $\times 4$ ' images of the extragalactic sky from CCAT SWCam (simulated; rms $=0.1 \mathrm{mJy}$ ), JCMT SCUBA-2 (actual data; $\mathrm{rms}=4.1 \mathrm{mJy}^{3}$ ), and Herschel SPIRE (actual data; $\mathrm{rms}=1.3 \mathrm{mJy}^{4}$ ). The CCAT and JCMT images are at the depth SWCam and SCUBA-2 could reach over 1 square degree in $\sim 1000 \mathrm{~h}$. The Herschel sources detected at $>5 \sigma$ are marked with orange circles in all images. These images illustrate that CCAT will have the sensitivity and angular resolution to resolve the cosmic far-infrared background and measure the properties of individual galaxies on scales and flux levels that are impossible with existing or planned far-infrared observatories

\section{SCIENCE GOALS MAPPED INTO SWCAM REQUIREMENTS}

\subsection{Selection of Colors}

The primary science driver for the CCAT observatory is to measure the bolometric luminosities and star formation rates of star-forming galaxies as a function of redshift. We chose our bands to map out the dust spectral energy distributions (SEDs) for distant star forming galaxies thereby providing rough photometric redshifts, and far-IR luminosities. The dust SEDs of star forming galaxies typically peak at rest wavelengths between 50 and $150 \mu \mathrm{m}$, corresponding to dust temperatures of $\sim 60$ to $20 \mathrm{~K}$. This peak, which traces the luminosity, arrives at our telescopes redshifted by the cosmic expansion and can be observed through the 350,450 and $850 \mu \mathrm{m}$ telluric windows for sources at redshifts from $\sim 1$ to 10. This redshift interval covers most of the star formation history of the Universe, beginning at only $\sim 500$ million

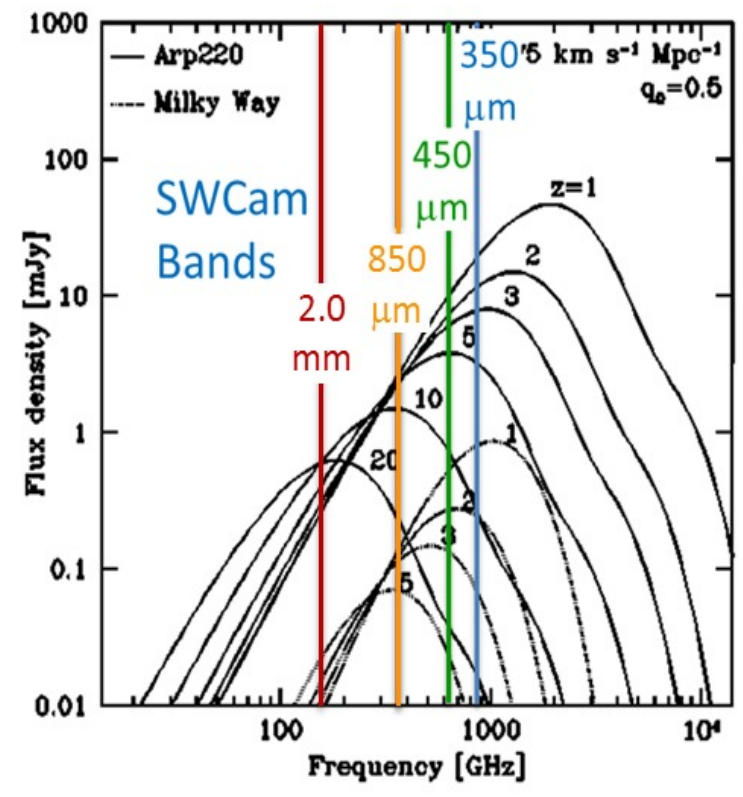

Figure 2. Far-IR/submm SEDs of the Milky Way and ULIRG galaxy Arp 220 as they would appear at various redshifts. The peak of the dust SED traces far-IR luminosity so that the four bands of SWCam trace submm SEDs and far-IR luminosities of galaxies over much of cosmic history from $\mathrm{z}$ $\sim 2$ to $>10$. The ULIRG ( $\mathrm{L}_{\text {far- }}$ $\left.\mathrm{IR}^{\sim} 1.5 \times 10^{12} \mathrm{~L}_{\odot}\right)$ Arp 220 would have a flux density $\sim 2$ $\mathrm{mJy}$ at $350 \mu \mathrm{m}$ if it were at $\mathrm{z}=$ 6. This is 10 times the confusion noise $(\sim 0.2 \mathrm{mJy})$ at $350 \mu \mathrm{m}$ so that it is easily distinguished. Note that due to the "negative K-correction" the $850 \mu \mathrm{m}$ flux is quite insensitive to redshift. years after the Big Bang $(\mathrm{z}$ 10) then integrating through the epoch of peak of star formation activity 2 to 3 Gyrs after the Big Bang ( $\mathrm{z}$ 3 to 2), and finishing 6 Gyrs after the Big Bang $(\mathrm{z} \sim 1)$ when the gradual decline in star formation activity to the current day levels was already well underway. The SWCam 350, 450, 850 and $2000 \mu \mathrm{m}$ bands are well tuned to deriving bolometric luminosities over these times (Figure 2). 

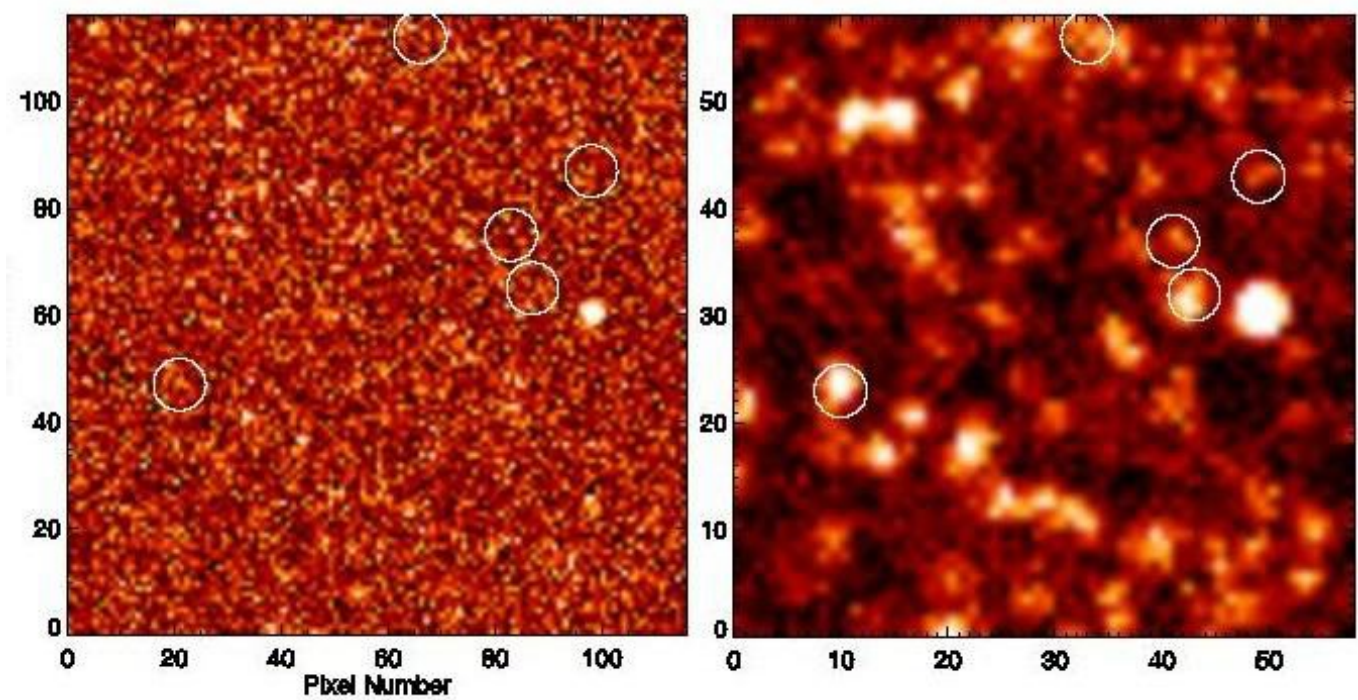

Figure 3. Simulated $350 \mu \mathrm{m}$ (left) and 850 $\mu \mathrm{m}$ (right) integrations with SWCam. The 5 circled sources are $>5$ $\sigma$ detections at $850 \mu \mathrm{m}$ that are not detected at $350 \mu \mathrm{m}$ - these are the high redshift galaxies that we seek.

We also will use source submm SEDs to quickly identify the highest redshift sources. As is clear from Figure 2, the 850 $\mu \mathrm{m}$ flux density of a given luminosity source is insensitive to redshift from redshift 1 to 5 . This is the familiar "inverse K-correction" whereby the dimming of a source due to distance is compensated by the brightening of the source as one rides further up the Rayleigh-Jeans tail of the dust SED. The inverse K correction makes $850 \mu \mathrm{m}$ galaxy surveys rapidly confusion limited, and rather eclectic with regards to source redshift. There is a similar, but less pronounced effect at other submm bands. For instance, the relatively weak K-correction at $350 \mu \mathrm{m}$ totally vanishes at redshifts beyond $\sim 3$ as the peak of the dust SED is redshifted beyond $350 \mu \mathrm{m}$ and the observed flux plummets down the Wien side of the dust SED. Therefore, high redshift sources will "drop-out" in $350 \mu \mathrm{m}$ surveys, so that $350 / 850 \mu \mathrm{m}$ comparisons will rapidly identify $\mathrm{z}>4$ sources (Figure 3). A similar effect occurs at $850 \mu \mathrm{m}$ and $2 \mathrm{~mm}$, where now the highest redshift $(\mathrm{z}>10)$ sources will drop-out in $850 \mu \mathrm{m}$ surveys, but be preserved in $2 \mathrm{~mm}$ surveys (Figure 2). These $850 \mu \mathrm{m}$ drop-outs are likely true proto-galaxy candidates.

\subsection{Maximum Beam Size.}

A small beam is required to break through source confusion, but we also need a small beam to identify individual sources, resolving them from close neighbors, and to obtain accurate positions for follow-up observations with CCAT spectrometers, ALMA, and other facilities. With its $25 \mathrm{~m}$ aperture, the diffraction- limited beam at $350 \mu \mathrm{m}$ is 3.5 ", which corresponds to $<30 \mathrm{kpc}$ - the size of the Milky Way galaxy - at all redshifts. We therefore will be separating Milky-Way sized systems at they appear throughout cosmic time. The high spatial resolution, large field imaging offered by CCAT promises to resolve the CIRB into its individual sources and separate the blends of sources in the larger beams of extant deep mm and submm-wave surveys (Figure 1).

The number of pixels allocated to each diffraction limited beam in the focal plane, also known as the spatial sampling, determines the effective beam size. The effective beam size is calculated by convolving the two dimensional Airy function with the pixel geometry as a function of offset between the two. We have performed the overlap integral for the $350 \mu \mathrm{m}$ beam as a function

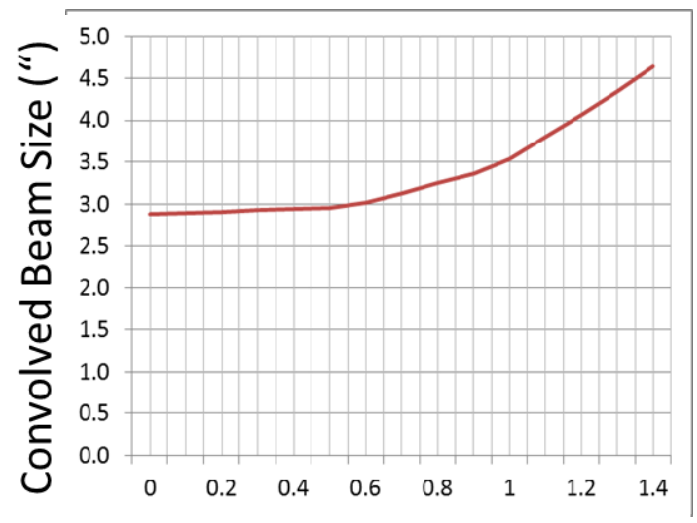

Pixel Size in Units of $\lambda / D$

Figure 4. Beam size as a function of spatial sampling. Pixel sizes are in units of $\lambda / \mathrm{D}=$ $2.9 "$ at $350 \mu \mathrm{m}$. of beam sampling (in units of $\lambda / \mathrm{D}$ ) (Figure 4). It is clear that sampling $\lambda / \mathrm{D}$ or finer will satisfy our requirement that the $350 \mu \mathrm{m}$ beam size be no larger than 3.5 " at $350 \mu \mathrm{m}$. 


\subsection{Mapping Speed}

For statistical analysis of the galaxy evolution over cosmic time, one would like to detect a large number (say 100,000 ) galaxies in each redshift interval (say $\Delta z \sim 0.25$ ) from redshift 1 to beyond 5 within a reasonable time-frame, say 5 years. To detect 4 (redshift bins) $\times 4$ (from $z=1$ to 5 ) $\times 100,000=1.6$ million galaxies at the confusion limit of 30 beams per source requires 48 million beams on the sky. We estimate the $350 \mu \mathrm{m}$ confusion limit is $1.04 \mathrm{mJy}$, so that with our 350 $\mu \mathrm{m}$ NEFD estimate of $13 \mathrm{mJy}-\mathrm{s}^{1 / 2}$ it will take 1.1 hours per beam for a $5 \sigma$ detection of the confusion limit. The entire survey therefore takes 52 million beam-hours on the sky. It is estimated that 888 hours/year will yield weather sufficiently good to deliver the $350 \mu \mathrm{m}$ sensitivity quoted above. We therefore require 12,000 beams at $350 \mu \mathrm{m}$ within our camera to complete such a survey in 5 years' time. We use pixels subtending $(\lambda / \mathrm{D})^{2}$ on the sky, and expect beams with FWHM $=1.22 \lambda / \mathrm{D}$. Therefore, to cover the equivalent field, 12,000 beams requires 17,500 pixels. Below we outline our design for a system with 2.74 times that number of beams at $350 \mu \mathrm{m}$, giving us a factor of 2.74 in observing time, or 1.66 in sensitivity safety factor for our science.

Mapping speed is coupled with spatial sampling. For a given spatial sampling, the mapping speed is proportional to the numbers of pixels in the camera. Pixel counts are a limiting commodity at present due to costs and the technical challenges involved in fielding ever larger format cameras, so that maximizing pixel counts within the cost/challenge envelope is not a well constrained problem. However, for a given number of pixels the mapping speed is a function of spatial sampling of the beam through the convolved beam size, so that the goal to maximize mapping speed will constrain our spatial sampling of the beam.

In the presence of source confusion, the mapping speed, in terms of the numbers of sources detected per unit time, is a function of the:

- Camera field of view: the number of pixels times the areal coverage per pixel

- Beam size: the convolution of the Airy pattern with the pixel size

- Point source sensitivity: this is insensitive to beam sampling until sampling size gets beyond $\sim 1.4 \lambda / D$, beyond which the noise increases more rapidly than signal in a background limited situation. At large values of sampling size, the point source sensitivity gets worse as the linear size of the beam, $\theta$, since the photon background is proportional to $\theta^{2}$.

- Confusion criterion: how many beams per source we assume for the confusion limit.

Under a given confusion criterion, the number of sources per FoV sampled by a beam is given by:

$$
N=\frac{\# \text { of beams }}{\text { beams } / \text { source }}=\frac{\text { field of view }}{\text { beam solid angle }} \div \frac{\text { beams }}{\text { source }}
$$

The number of sources detected per unit time is therefore a function of the beam sampling. As an example, suppose we have 47,600 square pixels in our $350 \mu \mathrm{m}$ array, each of which is 2.9 " on a side, so the sampling is $\lambda / \mathrm{D}$ at $350 \mu \mathrm{m}$. Then the field of view FoV has a total area of $400,300^{(") 2}$. The convolved beam size is $3.5^{\prime \prime}$ so that the FoV contains 32,700 beams. If the confusion limit is defined as 30 beams per source, then we would detect 1090 sources per FoV at the confusion limit.

However, with the same array, sampled with $1.45^{\prime \prime}$ square pixels $(\lambda /(2 \mathrm{D})$ at $350 \mu \mathrm{m})$ the FoV covers $100,080^{\left({ }^{(\prime) 2}\right.}$. The convolved beam is now 3.0" so that the FoV contains 11,100 beams. At the same confusion limit, we would detect 370 sources per FoV. The point source sensitivities are the same, so that per unit time, one detects $\sim 3$ times as many sources with the courser sampling.

We have calculated the number of sources detected per hour as a function of the beam sampling for a camera with 47,600 pixels at $350 \mu \mathrm{m}$ performing at twice the background limit ${ }^{\dagger}$ in the case of a 30 beams per source $5 \sigma$ confusion

\footnotetext{
${ }^{\dagger}$ We expect SWCam to be background limited. We will take data with SWCam by rapid scanning the telescope across the sky so that the (telluric) background fluctuations may be removed through a common mode analysis. The factor of 2 is included in the discussion here to be conservative, in case the sky subtraction is not totally lossless, adding noise to the final image.
} 
limit of $1.04 \mathrm{mJy}$. It is clear from Figure 5 that the detection rate rises rapidly to $\sim 215$ sources detected per hour within the 1.0 to $1.2 \lambda / \mathrm{D}$ sampling interval and drops off more slowly towards the coarser sampled regions of the plot. From the fine sampling end through $\sim 1.4 \lambda / \mathrm{D}$ sampling, the point source sensitivity is near uniform, but detection rates fall off steeply at finer sampling due to the reduced numbers of beams within a FoV (pixels are "wasted" for point source detection rates). The detections fall off more slowly at the more coarsely sampled regime due to a slow fall off in pointsource sensitivity

In the presence of noise intrinsic to the detector, the balance shifts markedly towards coarser sampling of the beam. Figure 6 shows the effects of injecting detector noise in quadrature with photon noise for total noise levels equal to 1.2 times and $1.4 \times$ the photon noise for a $\lambda / D$ sampled pixel (noise is injected at detection, then the total noise is multiplied by a factor of 2 as above). The detection rates go down dramatically for all pixel sizes, as one would expect. The rates go down most rapidly at the highly sampled (small pixel) regions of the plot (where detector noise is largest w.r.t. photon noise), and least rapidly for the most coarsely sampled (large pixel) regions of the plot where detector noise is smallest w.r.t. photon noise. If the detectors are not background limited, one should bias towards more coarsely sampled focal planes. However, CCAT science requirements demand that the $350 \mu \mathrm{m}$ beam size be no greater than 3.5 ", so that we cannot sample more coarsely than $\lambda / \mathrm{D}$ (Figure 4). The dual constraints (mapping speed and beam size) constrain our sampling to $\lambda / \mathrm{D}$ at $350 \mu \mathrm{m}$. Our arguments are not dependent on wavelength, so for consistency, we fix the sampling at $\lambda / \mathrm{D}$ for all four colors of the SWCam.

\subsection{Pixel Format and Physical Size}

We considered both square pixel and hexagonal pixel formats. We found that given the same pixel area, the convolved beam is more circular with hexagonal pixels, and marginally more compact. We also note that the centroids of hexagonal pixels are uniformly spaced, unlike a square format array, so that field sampling will be more uniform with hexagonal pixels. These are relatively minor issues. A technical issue, however, pushes us to select a hexagonal array format. For an LEKID, less than half the surface areas is the photo-sensitive meandering inductor. Therefore, in a filled array format, which is highly desirable from the optics point of view, we will need to have condensing optics (lenslets) in the focal plane to concentrate the light onto the meandering inductor. The condensing optics coupling becomes much more efficient in the near-circular hexagonal geometry.

Pixel size is driven by the competing needs of the optics and detector technology. For our $\lambda / \mathrm{D}$ sampling, the physical size of a pixel, $d$, is given by $d=f / \# \cdot \lambda$. Very small pixels (say $0.5 \mathrm{~mm}$ ) at $350 \mu \mathrm{m}$ require very steep $\mathrm{f} / \#$ 's (=1.4). Steep $\mathrm{f} / \#$ 's are very challenging for good optical quality over wide-fields. However, we would like to maximize the numbers of pixels on a monolithic array for ease of packing arrays into a focal plane driving us back to small pixel sizes. Pixel scales of $1 \mathrm{~mm}^{2}$ surface area are an excellent compromise between maximizing the numbers of pixels per monolithic array and keeping the optics straight-forward, and having sufficient surface area to design pixels with optical and electrical performance sufficient to yield background limited performance for both the $350 \mu \mathrm{m}$ and $450 \mu \mathrm{m}$ cameras. 


\section{SUB-CAMERA DESIGN}

\subsection{Choices}

The CCAT Nasmyth focus has an f-ratio of 6 , which would mean an $\mathrm{f} / \# \cdot \lambda$ pixel at $350 \mu \mathrm{m}$ is a reasonable $2.1 \mathrm{~mm}$ in physical size, so one may wonder why we do not directly image the CCAT focal plane onto our pixels. There is a compelling reason not to do this: reimaging to a smaller f/\# can provide an image of the secondary so that a cold Lyot stop may be inserted into the beam. Cold Lyot stops are a simple and important method for rejecting stray light and minimizing unnecessary thermal backgrounds. Without reimaging optics, it would be much more challenging for us to meet our sensitivity requirements.

We investigated several optical systems suitable for SWCam before arriving at our all transmission system outlined below. We find that an all reflective system will work, but requires large, off-axis optics that deliver marginal image quality over the $\sim 20$ ' FoV subtended by SWCam's final design. Furthermore, the all reflective system provides no easy way for implementing a cold Lyot stop. Finally, the CCAT project request co-mounting of instruments, which would be very difficult with all reflective optics.

\subsection{Sub-cameras and Lens Materials}

An on-axis transmissive design (lenses), can deliver excellent image quality in a very compact design. However, the f/6 CCAT focal plane is curved to the extent that diffraction limited imaging cannot be maintained over large fields without curving the focal plane. Clearly this is not practical for silicon lithography, so we will split the focal plane into individual sub-camera sections, each with a field lens at the appropriate position in the CCAT focal plane. Several optical design studies have demonstrated that diffraction limited imaging is possible over individual fields of view $>6$ ' in diameter at $350 \mu \mathrm{m}$ using planar arrays. To obtain the requisite 12' diameter FoV, at $350 \mu \mathrm{m}$, we split the focal plane into individual sub-cameras with 6' diameter fields of view: 4 sub-cameras at $350 \mu \mathrm{m}$, and 1 sub-cameras each at 450 $\mu \mathrm{m}, 850 \mu \mathrm{m}$ and $2 \mathrm{~mm}$. The 6 combined $350 \mu \mathrm{m}, 450 \mu \mathrm{m}$ and $2.0 \mathrm{~mm}$ cameras are symmetrically arranged around the central $850 \mu \mathrm{m}$ camera as shown in Figure 7.

A variety of materials are suitable for the lenses including HDPE and high purity silicon. These two materials are common and environmentally robust, and have modest loss tangents. However, since HDPE has an index $\mathrm{n}=1.5$ which is 2.27 times smaller than that of silicon, HDPE lenses must be 2.27 times thicker than silicon lenses to have the same refractive power. Therefore, even with similar loss tangents, the absorption losses in a HDPE lens can be significantly higher than those in a silicon lens. We have calculated the losses in the 3 element optical lens train (called out in Section 5 below) for both HDPE-based and silicon-based antireflection (A/R) coated lenses (reflection losses 1\% per surface). The worst case is for our $350 \mu \mathrm{m}$ system for which silicon-based lens train has $\sim 80 \%$ transmission, and the HDPE-based lens train has only $54 \%$ transmission - a factor of nearly 1.5 in favor of silicon. In addition, the $350 \mu \mathrm{m}$ emissivity of the warm field lens is $\sim 10 \%$ for the silicon-based system, and $\sim 30 \%$ for the HDPE-based system further degrading system
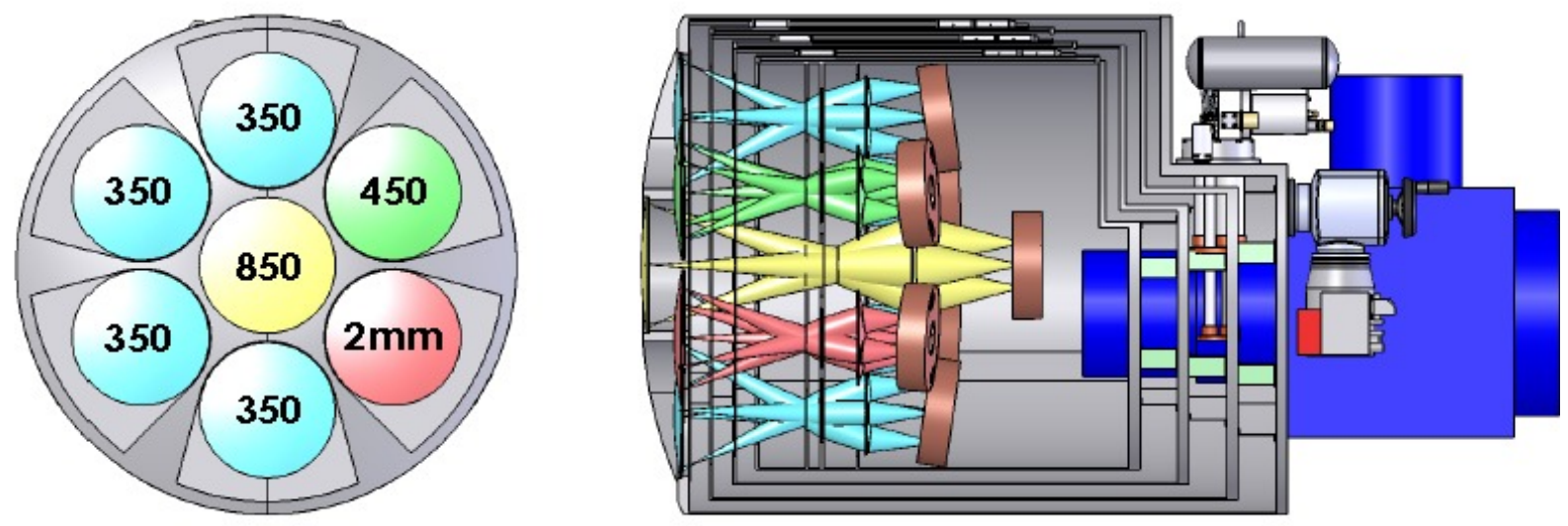

Figure 7. SWCam design overview. The right panel is a schematic SWCam side view (light enters from the left); the left panel shows the front view with its arrangement of sub-cameras. The optical elements are anti-reflection coated high purity silicon. 
performance for the HDPE-based system. Note that the transmission losses, and emissivity gains are much lower at the longer wavelengths for both systems but still silicon lenses remain superior to HDPE. Clearly a properly A/R coated silicon lens system is the best choice.

\subsection{Number of Sub-cameras per Color}

CCAT science is best enabled by four color mapping to scientifically relevant levels. We have seven sub-camera slots in our design. The question is how many slots to assign to each color. The standard mode of operation for large-scale deep surveys will be rapid scanning on the sky to enable demodulation and subtraction of the time variable sky backgrounds. Therefore, any given sub-camera will see the same integration time on the sky. Photon noise limited sensitivities differ at the four colors since the photon backgrounds are different, so one reasonable criterion might be to ask how long it takes to reach a given flux limit (mJy/beam) in each color. However, a far more relevant normalization is to the confusion limit at each color. In Table 1, we list the system sensitivity and our estimated confusion noise limits together with the mapping speed per sub-camera to $5 \sigma$ on these confusion limits for each wavelength. Our calculations (see Section 6 for the formulization) yield first quartile weather noise-equivalent flux densities (NEFD's) of 11.8, 8.1, and 3.7 and $1.7 \mathrm{mJy}_{-\mathrm{s}}{ }^{1 / 2} / \mathrm{beam}$ at $350,450,850 \mu \mathrm{m}$ and 2.0 respectively (Table 1). Confusion limits are not precisely established, but it is clear that a 4:1:1:1 (350:450:850:2000) ratio in sub-camera counts is well matched with the confusion limited mapping speed of $\sim 35^{(0) 2} /$ year in the 350,450 and $2000 \mu \mathrm{m}$ colors. The heavily negative K-correction favored $850 \mu \mathrm{m}$ band could map 3 times more area to the same limit. However, since all cameras operate simultaneously, our $850 \mu \mathrm{m}$ images will be $\sim \sqrt{3}$ deeper on the confusion than the others, i.e. to the $8.7 \sigma$ level on the confusion $(=1.03 / 8.66=0.12 \mathrm{mJy} /$ beam $)$.

\begin{tabular}{|c|c|c|c|c|c|c|c|}
\hline $\begin{array}{c}\text { Band } \\
\text { Center } \\
(\mu \mathrm{m})\end{array}$ & $\begin{array}{l}\text { Weather } \\
\text { Quartile }\end{array}$ & $\begin{array}{c}\text { Precipitable } \\
\text { Water } \\
\text { Vapor } \\
\end{array}$ & $\begin{array}{r}\text { Sensitivity } \\
\text { mJy-s } \mathrm{s}^{1 / 2} \\
\text { per beam }\end{array}$ & $\begin{array}{l}\text { Confusion } \\
\text { Limit(CL) } \\
\text { mJy/beam }\end{array}$ & $\begin{array}{c}\text { Sub-camera Mapping } \\
\text { Speed }\left({ }^{(0) 2} / \text { year }\right) \text { to } 5 \\
\sigma \text { on CL }\end{array}$ & $\begin{array}{c}\text { Sub- } \\
\text { cameras }\end{array}$ & $\begin{array}{c}\text { SWCam Mapping } \\
\text { Speed }\left({ }^{(\circ) 2} / \text { year }\right) \text { to } \\
5 \sigma \text { on CL }\end{array}$ \\
\hline 350 & $\begin{array}{l}1 \\
2 \\
3\end{array}$ & $\begin{array}{c}0.39 \\
0.71 \\
1.5\end{array}$ & $\begin{array}{l}11.8 \\
19.1 \\
53.8\end{array}$ & 1.04 & $\begin{array}{l}6.1 \\
2.3 \\
0.3\end{array}$ & 4 & 35 \\
\hline 450 & $\begin{array}{l}1 \\
2 \\
3\end{array}$ & $\begin{array}{c}0.39 \\
0.71 \\
1.5\end{array}$ & $\begin{array}{c}8.1 \\
13.3 \\
38.2\end{array}$ & 1.5 & $\begin{array}{c}27.2 \\
10.0 \\
1.2\end{array}$ & 1 & 38 \\
\hline 850 & $\begin{array}{l}1 \\
2 \\
3\end{array}$ & $\begin{array}{c}0.39 \\
0.71 \\
1.5\end{array}$ & $\begin{array}{l}3.7 \\
4.4 \\
6.7\end{array}$ & 1.03 & $\begin{array}{l}59.0 \\
41.6 \\
18.2\end{array}$ & 1 & 119 \\
\hline 2000 & $\begin{array}{l}1 \\
2 \\
3\end{array}$ & $\begin{array}{c}0.39 \\
0.71 \\
1.5\end{array}$ & $\begin{array}{l}1.7 \\
1.7 \\
1.9\end{array}$ & 0.23 & $\begin{array}{l}14.8 \\
14.1 \\
11.9\end{array}$ & 1 & 41 \\
\hline
\end{tabular}

\section{OPTICAL DESIGN}

Breaking the full field of view of SWCam into seven sub-cameras ensures good image quality at all wavelengths. We choose to put the $850 \mu \mathrm{m}$ camera on-axis so that it can serve as a test camera when first aligning the panels of CCAT during facility first light. We anticipate a long wavelength $(750$ to $3 \mathrm{~mm}$ ) camera (LWCam) will be installed on CCAT a few years after first light with SWCam. LWCam promises to be a much more capable instrument at $850 \mu \mathrm{m}$ and $2 \mathrm{~mm}$ than SWCam (due to its larger field of view at these wavelengths), so it is likely that after LWCam is fully operational, we will swap the $850 \mu \mathrm{m}$ core camera of SWCam with a $200 \mu \mathrm{m}$ core camera, and swap the $2.0 \mathrm{~mm}$ off-axis camera of SWCam with either an additional 350 or $450 \mu \mathrm{m}$ camera - the choice will reflect what we have learned about confusion limits in the first years of SWCam's use. To both make such changes easier, and to enable servicing of SWCam subcameras in the field, we require that each sub-camera have the same camera $\mathrm{f} / \#$ which translates into plate scale. We have fixed the $\mathrm{f} / \#$ at 2.86 so that the $\mathrm{f} \lambda$ pixel scale is $1.0,1.3,2.4$, and $5.7 \mathrm{~mm}$ at $350,450,850$, and $2000 \mu \mathrm{m}$ wavelength, respectively. We also hope to make entire sub-cameras modular, so that all that is required to change a subcamera is the disconnection of thermal and electrical attachments, and the withdrawal of the sub-camera "tube". 


\subsection{Optical Design.}

Our all $\mathrm{A} / \mathrm{R}$ coated silicon lens designs for the $850 \mu \mathrm{m}$ on-axis camera and the $350 \mu \mathrm{m}$ off-axis camera are illustrated in Figure 8. Every silicon lens in these designs is plano-convex, which simplifies lens manufacture and the application of $\mathrm{A} / \mathrm{R}$ coats. The individual lenses are identical for all cameras (save the $\mathrm{A} / \mathrm{R}$ coat). The only difference in the optical designs are that the pupil lens and the camera lens for the off-axis cameras are tilted $1^{\circ}$ with respect to the optical axis for the central pixel in each sub-camera field of view, and the focal plane array is tiled in the same sense by $8^{\circ}$. These small adjustments ensure very good image and pupil quality at all wavelengths.

Figure 9 shows a spot diagram for the $350 \mu \mathrm{m}$ camera over its field of view. Essentially all the power lies within the first null (circles) of the Airy pattern, and most within the full width-half maximum of the Airy pattern (half the circle radius). The high quality imaging is reflected, and quantified in Figure 10 which shows that the energy from point source that enclosed within a given radius from the center of a beam is virtually indistinguishable from that of an Airy function over the entire field of view. In fact, no pixel differs from the diffraction limit by more than $8 \%$ in terms of encircled energy at the $\lambda /(2 \mathrm{D})$ angular radius. The enclosed energy on a single hexagonal pixel with effective solid angle on the sky of $(\lambda / D)^{2}$ is $60 \%$. For the longer wavelength off-axis cameras, and for the on-axis $850 \mu \mathrm{m}$ cameras, the performance is even better. For further information on the optical design, including absorptive loss and optical/mechanical tolerances see Parsley ${ }^{7}$.
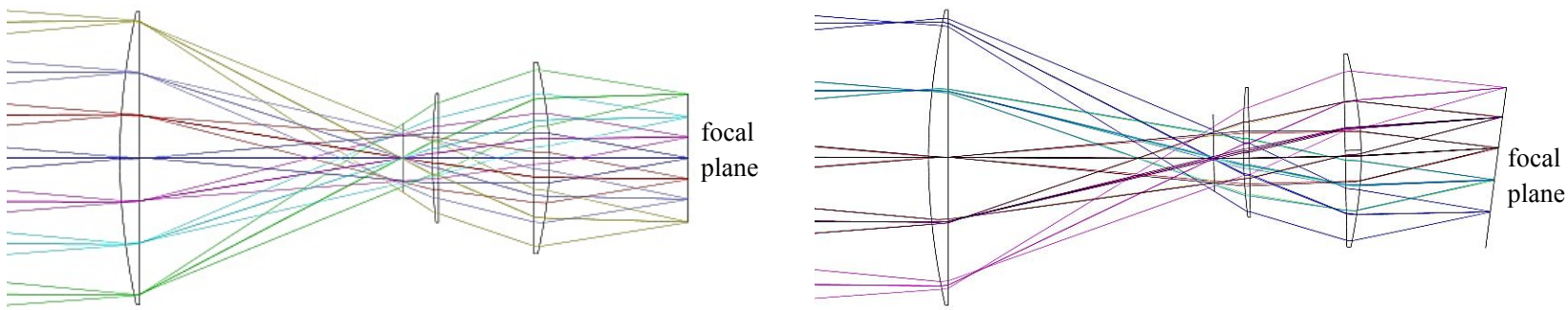

Figure 8. Optical ray traces for the (on-axis) $850 \mu \mathrm{m}$ core camera (left) and the $350 \mu \mathrm{m}$ off-axis camera (right). The optical elements and final f/\# each sub-camera (at all wavelengths) are identical. From left to right are the field lens (which also serves as the cryostat pressure window), pupil lens, camera lens, and focal plane.

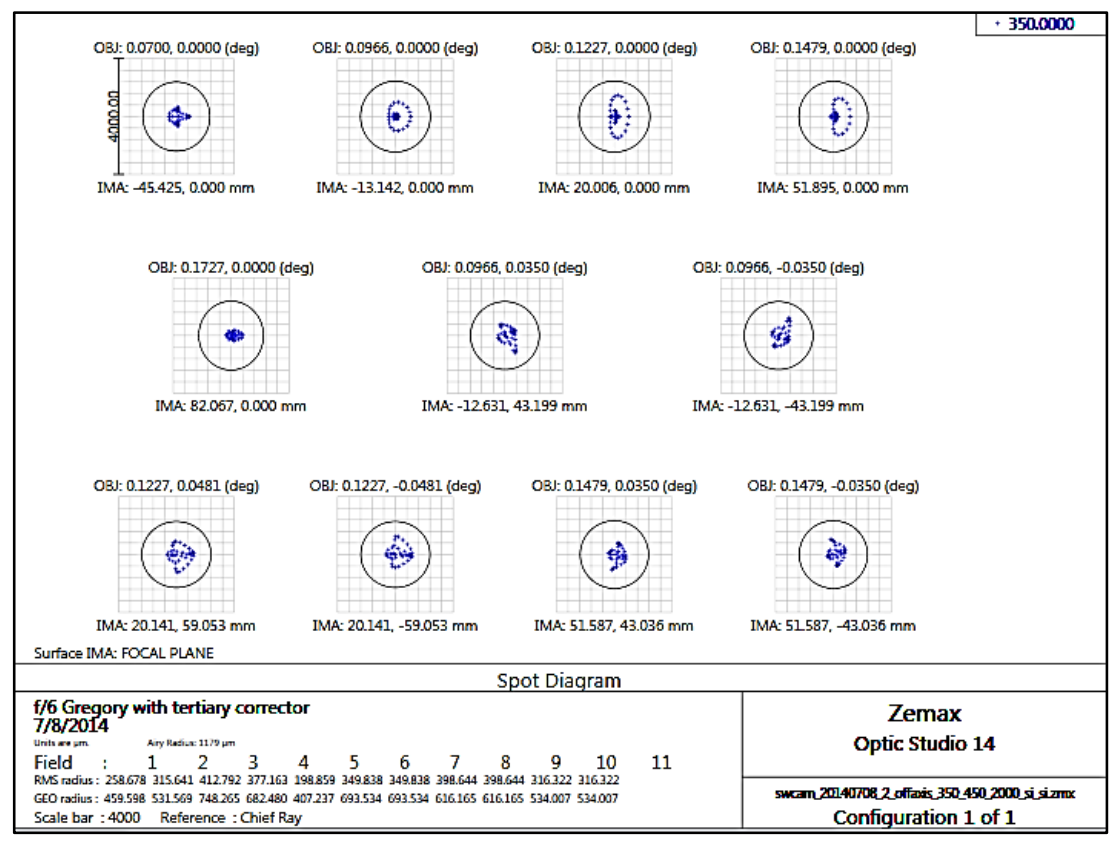

Figure 9. ZEMAX spot diagrams for the off-axis $350 \mu \mathrm{m}$ sub-cameras. Circles are the first null of the Airy pattern (diameter $=2.44 \lambda / \mathrm{D})$. The central pixel for this camera is 7.4' off the optical axis of the CCAT telescope. The spot diagram in row 1 , column 3 is for this central beam. Spot diagrams at column 1, row 1 and row 2 are for beams at the extremes of the 6' sub-camera field of view. 


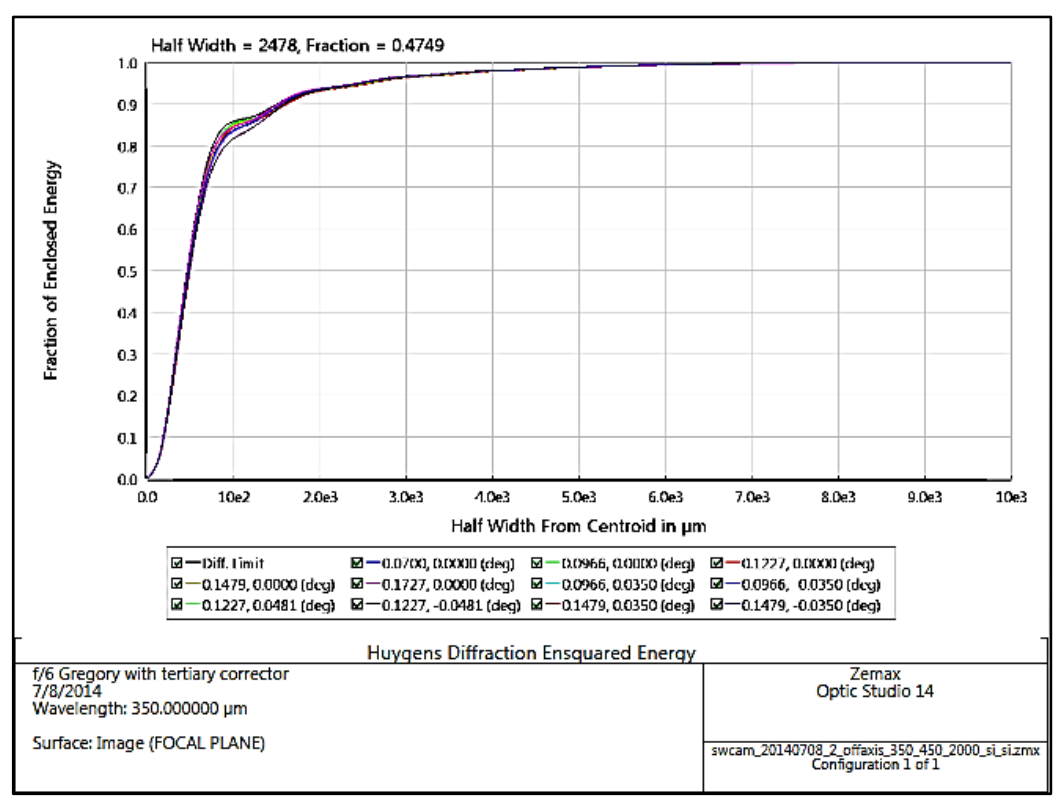

Figure 10. ZEMAX ensquared energy diagram for the $350 \mu \mathrm{m}$ sub-cameras generated using a Huygens diffraction approximation. The encircled energy is plotted as a function of offset from the center of the beam pattern for beams from the 11 positions of the spot diagram, and for a diffraction limited beam as comparison.

\subsection{Antireflection Coating of Optics}

Silicon has a high index of refraction $(\mathrm{n} \sim 3.4)$ which is good, and bad. It means that silicon lenses can be thin, yet still highly powered, so that extinction losses through the material are small, but it also means that there are very substantial reflection losses on the surface if it is not $\mathrm{A} / \mathrm{R}$ coated in some way. We have established a requirement that the reflective loss be less than $1 \%$ at each surface. The shorter wavelength bands have fractional bandwidth less than $12 \%$, so simple $1 / 4$ wave coatings would suffice for these, but a second order $\mathrm{A} / \mathrm{R}$ coat is in order for the $30 \%$ wide $2.0 \mathrm{~mm}$ bandpass. Tolerances involved in a $1 / 4$ wave coat at $350 \mu \mathrm{m}$ are $\sim 5 \mu \mathrm{m}(\sim \lambda / 20$ in the material). Therefore, the relatively simple methods of attaching $\mathrm{A} / \mathrm{R}$ coats such as gluing a layer of Cirlex onto the lens, then then machining this layer down to make a $1 / 4$ wave coating are not easily achieved. At the shorter wavelengths, it is possible to get a reasonably good $\mathrm{A} / \mathrm{R}$ coat by depositing parylene $1 / 4$ wave surfaces. However, the refractive index of parylene $(n=1.62)$ is not a perfect match for such coating $\left(\sqrt{ } n_{\text {silicon }}=\sqrt{ } 3.4=1.84\right)$, so even neglecting absorption losses (which may be as bad as $4 \%$ per surface), the best we can hope for with a $1 / 4$ wave parylene $\mathrm{A} / \mathrm{R}$ coat is an average transmission across our band of $98 \%$. The $>2 \%$ loss is outside of our $<1 \%$ across the band requirement (Figure 11 (left)), so simple parylene coats are not sufficient.

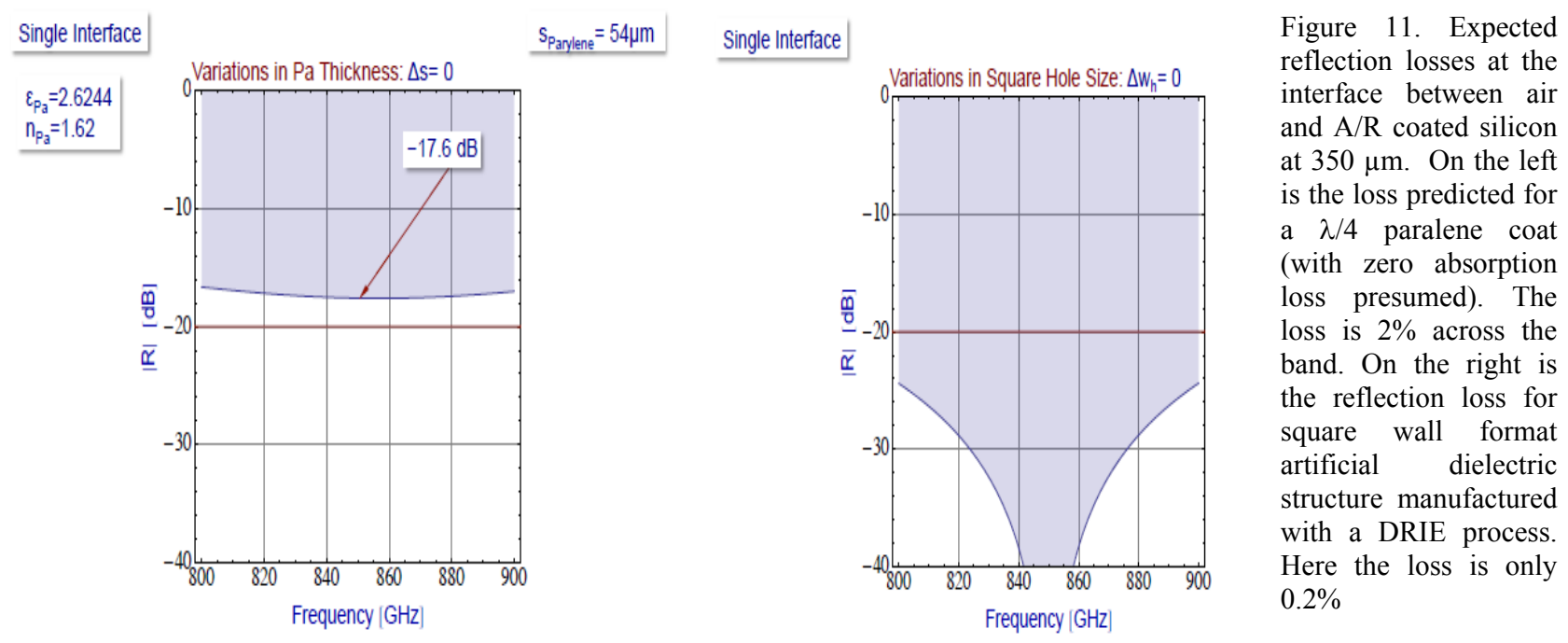


We have undertaken programs to create artificial dielectric structures through cutting holes or grooves in the surface of the silicon itself. If a suitable amount of material is removed, and the size-scale of structures is small compared with the wavelength of interest, then a surface layer of the desired $V_{n}$ index may be created resulting in a very high efficiency $\mathrm{A} / \mathrm{R}$ coating (Figure 11, right). The wavelength of the $\mathrm{A} / \mathrm{R}$ coat is tuned by making the depth of the structures $\lambda / 4$ in the medium. We have pursued such engineered $\mathrm{A} / \mathrm{R}$ coats through both mechanical means (a dicing saw) and deep reactive ion etch (DRIE) processing.

Both techniques are viable, but both have their drawbacks. The dicing saw method is slow - it likely will take one to two months per surface to $\mathrm{A} / \mathrm{R}$ coat a $350 \mu \mathrm{m}$ lens. The DRIE process is much faster, but unfortunately, DRIE facilities cannot coat silicon wafers (or lenses) that are more than a few mm thick. Therefore, we would need to DRIE the A/R surface on a thin $(\sim$ few tenths of mm thick) silicon wafer and then bond that wafer to the actual lens surface. For the planar side this attachment has been demonstrated by others, and is straightforward. However, for the convex side, we will have to first bend, then bond, which has yet to be demonstrated. That said, we have created both dicing saw and DRIE surfaces on thin wafers, and our first transmission tests are very encouraging. For silicon surfaces coated on a single side, the measured transmission profiles match our modeling within our measurement errors for both types of structured coatings. We are preparing thin silicon wafers with double sided DRIE, double sided parylene, and one-sided DRIE/one side parylene A/R coatings, and will measure their transmission processes with an FTS. It is expected that the double sided measurements will give a much more accurate measurement of the actual transmissions. The one-side DRIE/one-sided parylene $\mathrm{A} / \mathrm{R}$ coat is being tested as a fall-back solution in the case we cannot bend and attach a thin DRIE A/R coat to the curved surfaces of our lenses. In this case, we might create a DRIE surface (with very low reflectivity) on the flat side of our plano-convex lens and parylene on the curved side, thereby achieving our net $1 \%$ loss per surface requirement (if there is no bulk absorption in parylene). More information on the dicing saw and DRIE A/R coating structures we are developing can be found in and Wheeler ${ }^{8}$ and Koopman 9

\subsection{Filter Parameters}

The background power from the sky within a SWCam pixel is quite modest: $\sim 20,20,100$, and $130 \mathrm{pW}$ at $2.0 \mathrm{~mm}, 850$, 450 , and $350 \mu \mathrm{m}$ respectively, much smaller than the blackbody radiation emitted by $1 \mathrm{~cm}^{2}$ of $4 \mathrm{~K}$ surface, so care is taken for optical filtration. Fortunately, many very high quality submillimeter filters have been developed by P. Ade and C. Tucker's group at Cardiff University. We take advantage of these commercially available filters to construct a robust, yet highly transmissive filtration chain. As an illustrative example, we show the filters, their parameters and the temperature stage they will be mounted for the $350 \mu \mathrm{m}$ camera in Figure 12. Most of the power from room temperature radiation is rejected at the dewar entrance by a series of four lithographed metal "thermal" filters attached in series to the $300 \mathrm{~K}, 2 @ 80 \mathrm{~K}$, and $15 \mathrm{~K}$ shields. Behind the thermal shields there are a series of long wavelength pass (LP) filters two on the $4 \mathrm{~K}$ shields (the first is $56 \mathrm{~cm}-1 \mathrm{LP}$, and the second $35 \mathrm{~cm}-1 \mathrm{LP}$ ) and the final one (again, a $35 \mathrm{~cm}-1 \mathrm{LP}$ filter) is on the $1 \mathrm{~K}$ shield. Finally, there is a bandpass (BP) filter just in front of the detector array serving as the entrance window to the $100 \mathrm{mK}$ detector house. This last filter sets the detector optical bandpass, and blocks long wavelength

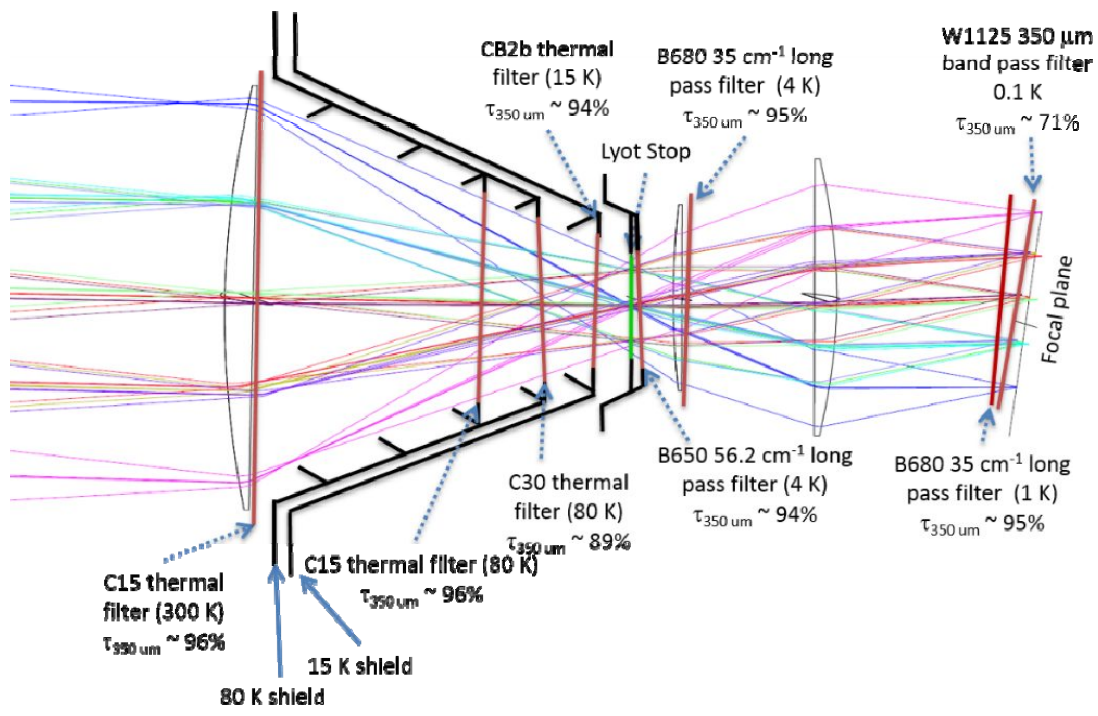

Figure 12. Baffles and filter locations for the $350 \mu \mathrm{m}$ sub-cameras as described in the text. 
radiation from cold $(4 \mathrm{~K})$ surfaces within the dewar. The series of filters is available in the desired sizes from the Cardiff group at reasonable cost. The total transmission of the $350 \mu \mathrm{m}$ filtration chain is a respectable $47 \%$.

The field lens serves as the entrance window to the dewar, and is therefore at ambient temperature. The Lyot lens in the 350 and $450 \mu \mathrm{m}$ cameras is held at $4 \mathrm{~K}$ and with its low emissivity and temperature it does not add to the background radiation within the beam. The cameras lens is also held at $4 \mathrm{~K}$. The field lens counts as a warm transmission item and the other two lenses count as part of the cold optical transmission.

\section{SENSITIVITY ESTIMATES}

Referred to the detector, the noise equivalent power, NEP, of a background-limited camera with warm optics at temperature $\mathrm{T}$, and emissivity $\varepsilon_{\text {warm }}$, cold optical elements of transmission $\eta_{\text {cold }}$ (emissivity 0 ) and detector quantum efficiency $\eta_{\text {det }}$, is given by:

$$
\mathrm{NEP}=h v\left\{\Delta v \cdot \mathrm{N} \cdot \mathrm{p} \cdot \varepsilon_{\text {warm }} \eta_{\text {det }} \cdot \eta_{\text {cold }} \tilde{\mathrm{n}}\left(1+\varepsilon_{\text {warm }} \eta_{\text {det }} \eta_{\text {cold }} \tilde{\mathrm{n}}\right) \cdot 2\right\}^{1 / 2} \text { Watts } \mathrm{Hz}^{-1 / 2}
$$

Where:

- $\quad h$ is Planck's constant, $v$ is frequency, $\Delta v$ is bandwidth,

- $\quad \mathrm{N} \equiv \mathrm{A} \Omega / \lambda^{2}$ is the number of spatial modes (=0.8 for 2.9" pixels @ $\left.350 \mu \mathrm{m}\right)$

- $\mathrm{p}$ is the number of polarization modes accepted by the detector

- $\tilde{\mathrm{n}}=1 /(\exp (\mathrm{h} v / \mathrm{kT})-1)$ is the mode occupation number $(=5.8 @ 350 \mu \mathrm{m}$ for $\mathrm{T}=260 \mathrm{~K})$

- The factor of 2 is from expressing the NEP in $\mathrm{Hz}^{-1 / 2}$

- $\eta_{\text {cold }} \sim 40 \%$ is the product of all the blocking filters and cold optics

- We take the detector quantum efficiency, $\eta \sim 75 \%$

- $\varepsilon_{\text {warm }}$ includes both the sky and telescope contributions: $\varepsilon_{\text {warm }}=\tau_{\text {Field Lens }} \cdot\left(\left(1-\eta_{\text {sky }}\right) \cdot \eta_{\text {tel }}+\left(1-\eta_{\text {tel }}\right)+\varepsilon_{\text {Field Lens }}\right.$, where:

- $\tau_{\text {Field Lens }}$ is the transmission of the field lens;

- $\eta_{\text {sky }}$ is the zenith transparency of the sky;

- $\eta_{\text {tel }} \sim 90 \%$ is the forward efficiency of the telescope (losses are essentially Ohmic);

$\circ \varepsilon_{\text {Field Lens }}$ is the emissivity of the field lens, which serves as the entrance window

We assume that the intrinsic noise in the detectors themselves is small compared to the photon noise, so that we are background limited.

The NEP is related to the noise-equivalent flux density (NEFD) on the sky by:

$$
\mathrm{NEFD}=\mathrm{NEP} /\left(\mathrm{A}_{\text {tel }} \cdot \Delta v \cdot \eta_{\text {det }} \cdot \eta_{\text {cold }} \cdot \eta_{\text {Field Lens }} \cdot \eta_{\text {tel }} \cdot \eta_{\text {spill-over }} \cdot \eta_{\text {Ruze }} \cdot \eta_{\text {sky }}\right)
$$

Where:

- $\mathrm{A}_{\mathrm{tel}}$ is the geometric area of the telescope primary

- $\Delta v$ is the detection bandwidth, determined by the band-pass filter

- $\eta_{\text {Field Lens }}=$ the transmission of the field lens

- $\eta_{\text {spill-over }}$ is the spill-over efficiency past the secondary (estimated at $90 \%$ )

- $\eta_{\text {Ruze }}=\exp \left(-(4 \pi \cdot \delta / \lambda)^{2}\right)$ is the Ruze efficiency due to small-scale surface errors of the optical system where the telescope specification is $\delta=12.5 \mu \mathrm{m}$ rms.

The NEP and NEFD discussed above refer to a single pixel. Single pixel NEFDs are useful for extended source calculations, where the source fills many pixels in the focal plane. To refer the NEFD to a beam, however, we must take into account the fraction of the light from a point source that is captured by a single pixel. For our hexagonal pixels subtending $(\lambda / \mathrm{D})^{2}$ sr on the sky, the fraction of the power from a point source captured by a single pixel is $60 \%$. Point source sensitivities are therefore per pixel sensitivities divided by 0.6. Table 1 above lists sensitivity estimates for SWCam in its four bands referred to a beam. To make the calculation, we use the weather statistics from Radford ${ }^{5}$. 


\section{LEKID ARRAYS}

To fulfill our science requirements we need approximately a few tens of thousands of $350 \mu \mathrm{m}$ pixels working at, or very near to the background limit in our focal plane. While time-domain SQUID multiplexed TES detectors have demonstrated the requisite sensitivity, the MUX factor for such systems is only $\sim 32$. This complicates electronics and thermal issues within and outside the cryostat, so that existing SQUID muxed TES arrays have reached their practical limits within the SCUBA-2 system at $\sim 5200$ pixels per color in a camera. Our 57,160 pixel camera would be quite challenging to construct and implement with this technology. Fortunately, Lumped Element Kinetic Inductance Detectors (LEKID) arrays are coming to maturity in their development at JPL and other labs in the world. These arrays promise background limited performance with high detective quantum efficiency and very high MUX factors - current systems at JPL are reading out more than 500 pixels per line.

LEKIDs are detectors in which photon energies are directly absorbed into a meandering superconducting inductor that is part of a superconducting resonant circuit. The absorbed photon energy breaks Cooper pairs in the superconducting film, increasing quasi-particle numbers, hence the reactance of the inductor. The change in frequency of the resonant circuit is detected in a transmission-line readout as the detector response to incident photons. The (unique) signal from each pixel is confined to a limited band-width so that with a large bandwidth in the transmission line, many $(\sim 1000)$ pixels can be read out with a single 50 to $250 \mathrm{MHz}$ band-width transmission line.

TiN LEKID development for SWCam is part of an ongoing broader effort at JPL to bring the KID technology to full fruition. The development work has been tested in the lab, validating the advantages inherent in the KID technology including relative ease of manufacture, and high multiplex factor and pixel yield, clean readout noise spectra with low cross-pixel coupling, and no unexpected systematics. In April 2013, a proto-type camera, MAKO, that contained 432 TiN LEKIDs operating in the $350 \mu \mathrm{m}$ telluric window was field tested at the CSO with good success (Figure 13). This first test involved a single polarization array with no condensing optics. Even so, it was within a factor of 3 to 4 of background limited performance (BLIP). Since then, optical coupling has been greatly improved through the addition of parylene $\mathrm{A} / \mathrm{R}$ coated lenslet arrays, and the dominant source of noise, two level system noise, has been overcome through enlarging the capacitor and shrinking the inductor in the resonant circuit. This $2^{\text {nd }}$ generation MAKO device appears to be near the background limit in the lab (design shown in Figure 14). The next generation MAKO device (Gen 3 ) introduces a complex meandered inductor that is sensitive to both polarizations, so that this should increase the net sensitivity by up to a factor of $\sqrt{ } 2$ over the previous generation devices. It is expected that the dual polarization device will detect $\sim 75 \%$ if the light incident on a pixel. First tests in the lab are complete, and the Gen 3 device is intended to be taken to the telescope in late August 2014 for an engineering run with MAKO. For more information on the LEKID developments at JPL see McKenney ${ }^{10}$.

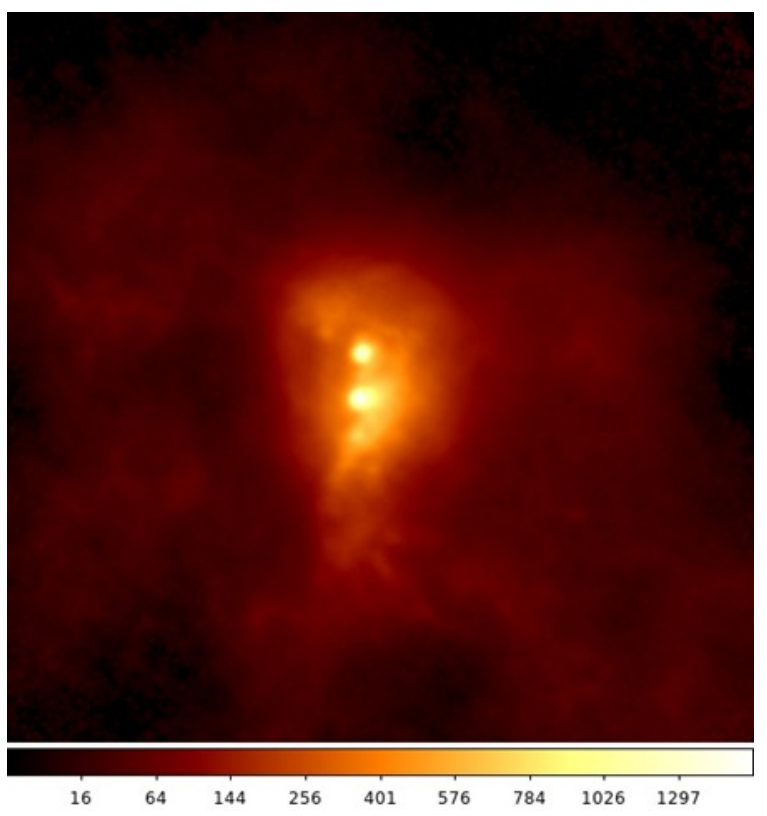

Figure 13 (left). MAKO $350 \mu \mathrm{m}$ image of Sgr B2 obtain at first light on the CSO (Swenson et al in preparation).

Figure 14 (below). Layout showing inductors (central circular feature) capacitors (large red-rectangles), readout line (thick red line) and lens positions (black) of hexagonal Gen 2 array.

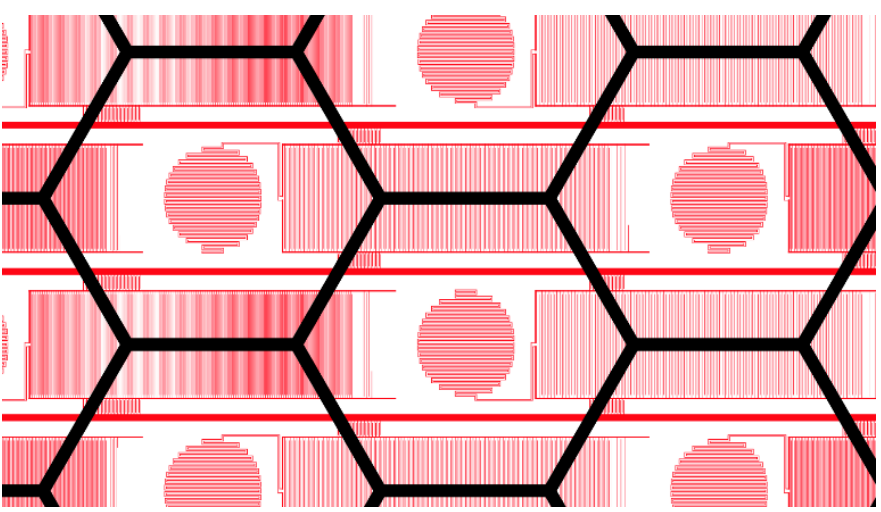




\section{READOUT ELECTRONICS}

The beauty of LEKID arrays is that each pixel in an array can be manufactured to have its own unique resonant frequency so that it responds uniquely to a tone sent down a transmission line. If each resonant tone has sufficient $\mathrm{Q}$ to be separated from its neighbors then a large number of tones may be read out on a single line. The Caltech/MAKO 350 $\mu \mathrm{m}$ camera development has pushed the readout technology along quite rapidly. The current plan is to read out $\sim 1000$ pixels along a single transmission line that operates in the $\sim 50$ to $250 \mathrm{MHz}$ band. At present, MAKO has demonstrated readouts of $\sim 500$ pixels on a single line, so that the goal of 1000 appears readily obtainable.

Sending in the tones, and then receiving and analyzing the response is a non-trivial, but straightforward application of modern high speed digital electronics. MAKO read out the tones from its array at the CSO using a Roach-based system and it is also possible to read-out with a GPU-based FFT engine. The latter is required if one plans to read out the arrays by sending in a "chirp" signal that excite all resonant frequencies. The chirp based readout has the advantage over a tone-based readout in that it has higher dynamic range for input signals, and has much more constant sensitivity as observing conditions change. For more information on the readout electronics see Rajagopan ${ }^{11}$ and Kovacs ${ }^{12}$.

\section{CRYOSTAT}

The SWCam cryostat is a welded aluminum cylinder about 2 meters long and 1.3 meters in diameter. There are 5 cooling stages. Two dual stage pulse tube coolers from Cyomech, Inc. provide cooling at 80, 15, and $4 \mathrm{~K}$, while a dilution refrigerator provides cooling at 1 and $0.1 \mathrm{~K}$ (BlueFors Cryogenics Oy Ltd.) Details of the design (design requirements, and their links to, for example, the thermal budget, thermal links, filtration, optics mounts) are found in Parshley ${ }^{7}$. The requirements for the cryostat are driven by detector operating parameters, optical layout (ray trace, size of optical elements, stray light mitigation, filters), and, of course the telescope environment (available resources, ambient conditions, project standards). A section view of the cryostat is shown in Figure 15 detailing the shields and temperature stages as well as the location of the cryogenic and vacuum systems at the rear of the cryostat.

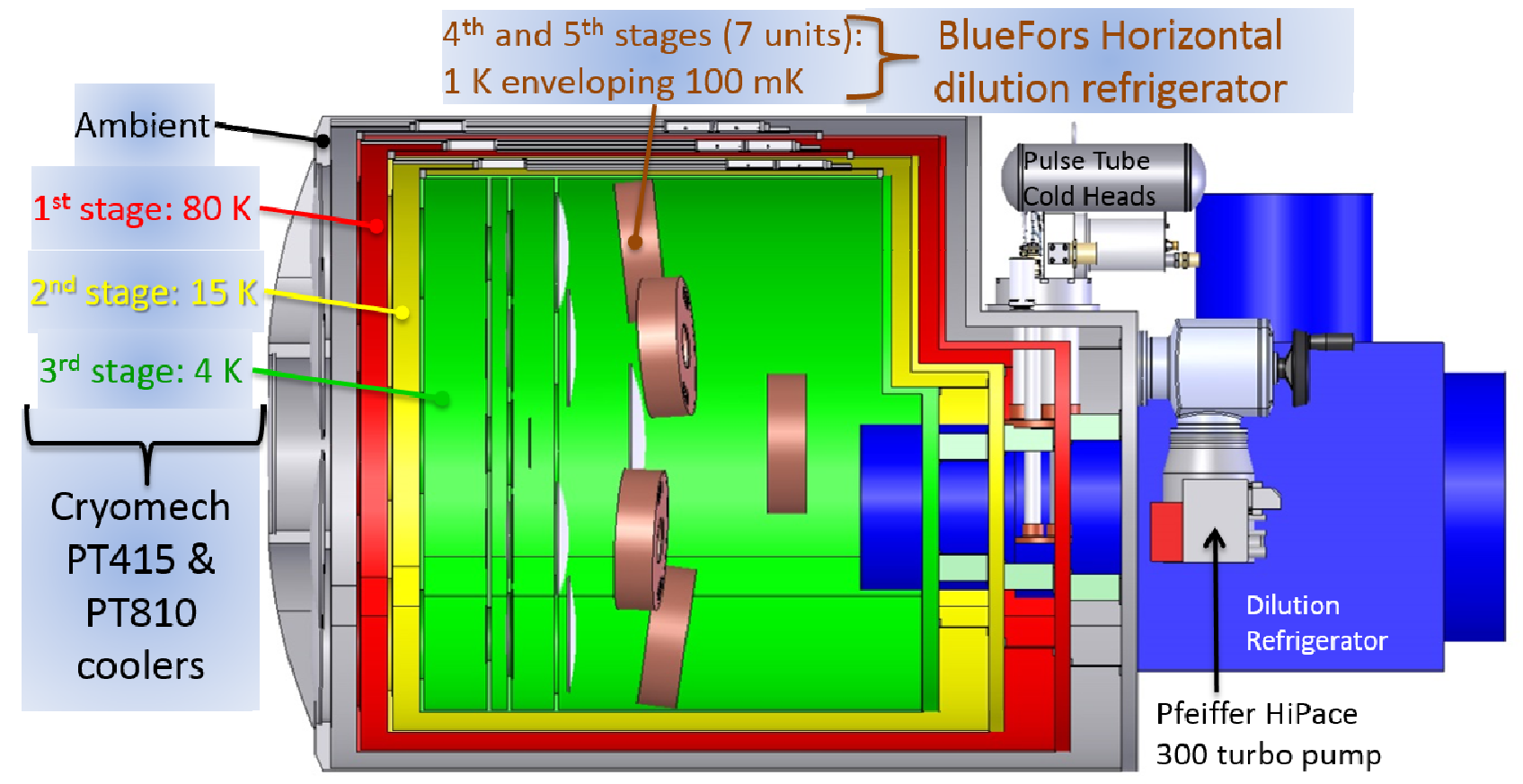

Figure 15. Schematic of the SWCam cryostat showing locations and sources of various temperature stages. Light enters from the left. 


\section{COMPARISON WITH OTHER COEVAL FACILITIES}

It is instructive to compare the capabilities of SWCam on CCAT to those of other coeval facilities such as the CSO, JCMT and ALMA. We do this by comparing existing instrumentation, but also note the sensitivity one would expect for SWCam on the CSO and JCMT as a sanity check. If a given instrument is put on a variety of telescopes, the relative point source sensitivity is directly proportional to the ratios of geometric collecting areas of the telescope, the Ruze factors (telescope efficiency as a function of wavelength) and the zenith transmission at the sites, and inversely proportional to the NEP (which is a soft function of site at 350 and $450 \mu \mathrm{m}$ since emissivities are high). The important parameters for the SHARC-II camera on CSO, the SCUBA-2 camera on JCMT and ALMA (excepting NEP) are listed in Table 2 for comparison. We use top quartile values for water vapor burden in all cases (note that for our SWCam/CCAT sensitivities, we used the top third value for PWV $(=0.43 \mathrm{~mm}))$. As an example, the ratios for CCAT to CSO are: $\left(\mathrm{A}_{\mathrm{CCAT}} / \mathrm{A}_{\mathrm{CSO}}\right)=5.7 ;\left(\eta_{\text {ruze, }}\right.$ CCAT $\left.\left./ \eta_{\mathrm{Ruz}-\mathrm{CSO}}\right)=1.2 ; \eta_{\text {sky, } \mathrm{CCAT}} / \eta_{\text {sky-CSO}}\right)=2.0$. Therefore, if SWCam were installed on the $\mathrm{CSO}$, one would expect a point source sensitivity of $26 \mathrm{mJy} \times 14=350 \mathrm{mJy} \mathrm{sec}^{1 / 2}$ (column 8 in Table 2) - the same as the observed value for SHARC-II/CSO. This solid agreement adds confidence to our calculations. Our prediction is that SWCam would be substantially more sensitive than SCUBA 2 at $450 \mu \mathrm{m}$, and about 1.5 times more sensitive at $850 \mu \mathrm{m}$ if both were operated on JCMT. The last column in Table 2 is the point source sensitivity gain that the SWCam/CCAT combination will have over comparison facilities. For example, SWCam/CCAT will be 14 times more sensitive than SHARC-II/CSO at $350 \mu \mathrm{m}$, and 9 times more sensitive than SCUBA-2/JCMT at $850 \mu \mathrm{m}$. Finally, SWCam/CCAT is equal in sensitivity to the 50 element ALMA array at $350 \mu \mathrm{m}$, and only a factor 0.6 worse that ALMA at $450 \mu \mathrm{m}$. This perhaps surprising result is actually quite easy to understand. The geometric area of $12 \mathrm{~m}$ CCAT dish is equivalent to 4.3 - $12 \mathrm{~m}$ ALMA antennas. At $350 \mu \mathrm{m}$, the surface roughness (CCAT $=12.5 \mu \mathrm{m}$; ALMA $=25 \mu \mathrm{m}$ ) wins a factor of 1.9 (Ruze factor) for CCAT, the $97 \mathrm{GHz}$ bandwidth of dual polarization CCAT detectors wins a factor of $(97 / 8)^{1 / 2}=3.5$ for CCAT, the Chajnantor site wins a factor of 1.35 for CCAT (comparing top quartiles), and the intrinsic sensitivity advantage of direct detection over heterodyne detection combined with sky emissivity wins another factor of 2 over ALMA. Therefore, for point source detection at $350 \mu \mathrm{m}$, CCAT sensitivity should be equivalent to $~ 62$ ALMA antennas combined. The ALMA online sensitivity calculator values (listed in Table 2) confirm this line of logic.

\begin{tabular}{|c|c|c|c|c|c|c|c|c|}
\hline $\begin{array}{l}\text { Facility and } \\
\text { Aperture(s) }\end{array}$ & $\begin{array}{c}\text { Surface } \\
\text { Roughness } \\
\text { (rms) }\end{array}$ & Band & $\eta_{\text {Ruze }} 1$ & $\mathbf{P W V}^{2}$ & $\eta_{\text {sky }}{ }^{3}$ & $\begin{array}{l}\text { Sensitivity }^{4} \\
\left(\mathrm{mJy} \mathrm{sec}^{1 / 2}\right)\end{array}$ & $\begin{array}{c}\text { SWCam } \\
\text { Sensitivity } \\
\text { on given } \\
\text { facility }^{5}\end{array}$ & $\begin{array}{c}\text { SWCam/ } \\
\text { CCAT } \\
\text { Sensitivity } \\
\text { Gain }^{6}\end{array}$ \\
\hline \multirow{3}{*}{$\begin{array}{c}\text { SWCam/CCAT } \\
D=25 \mathrm{~m}\end{array}$} & \multirow[t]{3}{*}{$12.5 \mu \mathrm{m}$} & 350 & $82 \%$ & \multirow[t]{3}{*}{0.38} & $55 \%$ & 26 & - & - \\
\hline & & 450 & $88 \%$ & & $64 \%$ & 18 & - & - \\
\hline & & 850 & $97 \%$ & & $91 \%$ & 8.4 & - & - \\
\hline $\begin{array}{c}\text { SHARC-II/CSO } \\
\text { D }=10.4 \mathrm{~m}\end{array}$ & $17 \mu \mathrm{m}$ & 350 & $69 \%$ & 0.85 & $28 \%$ & 350 & 350 & 14 \\
\hline \multirow{2}{*}{$\begin{array}{c}\text { SCUBA } \\
\text { 2/JCMT D }=15\end{array}$} & \multirow{2}{*}{$25 \mu \mathrm{m}$} & 450 & $61 \%$ & \multirow[t]{2}{*}{0.85} & $32 \%$ & 710 & 147 & 38 \\
\hline & & 850 & $87 \%$ & & $79 \%$ & 85 & 58 & 9 \\
\hline \multirow{3}{*}{$\begin{array}{c}\text { ALMA } \\
D=50 \times 12 \mathrm{~m}\end{array}$} & \multirow[t]{3}{*}{$25 \mu \mathrm{m}$} & 350 & & \multirow[t]{3}{*}{0.66} & $41 \%$ & 26.5 & - & 1 \\
\hline & & 450 & & & & 11.5 & - & 0.62 \\
\hline & & 850 & & & & 1.05 & - & 0.11 \\
\hline \multicolumn{9}{|c|}{$\begin{array}{l}{ }^{1} \text { Ruze efficiency. Note that the Ruze factors are not used for any calculations involving ALMA, so they are not tabulated. } \\
{ }^{2} \text { Top quartile PWV from S. Radford }{ }^{5} \text {. } \\
{ }^{3} \text { Band averaged sky transparency at zenith. } \\
{ }^{4} \text { mJy }(1 \sigma) \text { in } 1 \text { second integration time. From online calculators for SCUBA } 2 \text { and ALMA ( } 50 \text { antennas). SWCam/CCAT } \\
\text { estimates includes a factor of } 2 \text { degradation for imperfect sky subtraction (this is equivalent to the losses entailed by } \\
\text { the use of a chopping secondary). } \\
{ }^{5} \text { Predicted sensitivity (mJy sec }{ }^{1 / 2} \text { ) of SWCam on the other facility (for JCMT and CSO). } \\
{ }^{6} \text { Sensitivity ratio for SWCam/CCAT as compared with the other facility and its instrumentation. }\end{array}$} \\
\hline
\end{tabular}




\section{CONCLUSIONS}

SWCam is the critical instrument that enables CCAT to pursue its primary science: to trace the obscured star formation history of the Universe. We have completed a preliminary design study that demonstrates SWCam can deliver the key data, namely surveys in the $350,450,850$ and $2000 \mu \mathrm{m}$ bands over a few hundred square degrees of sky to the confusion limit in each band in 5 years' time. The key enabling technology for this are the 57,160 pixel TiN LEKIDs detector arrays, and their development is proceeding on schedule to enable a first light with SWCam on CCAT in the 2019 to 2020 time-frame.

Acknowledgements: This work was supported in part by the CCAT telescope project, NSF AST-118243.

\section{REFERENCES}

[1] Woody, D., Padin, S., Chauvin, E., Clavel, B., Cortes, G., Kissil, A., Lou, J., Rasmussen, P., Redding, D., Zolkower, J., "The CCAT 25m diameter submillimeter-wave telescope", Proc. SPIE, 8444, (2012).

[2] Fixsen, D. Dwek, E. Mather, J.C. Bennett, C.L., and Shafer, R.A., "The Spectrum of the Extragalactic Far-Infrared Background from the COBE FIRAS Observations", ApJ 508, 123 (1998).

[3] Casey, C. M., C.-C. Chen, L. L. Cowie, L. et al., "Characterization of SCUBA-2 $450 \mu \mathrm{m}$ and $850 \mu \mathrm{m}$ selected galaxies in the COSMOS field". MNRAS 436, 1919-1954, (2013).

[4] Oliver, S. J., J. Bock, B. Altieri, A. Amblard, V. et al., "The Herschel Multi-tiered Extragalactic Survey: HerMES". MNRAS 424, 1614-1635, (2012).

[5] Radford, S.J.E., "Observing Conditions for Submillimeter Astronomy. In Astronomical Site Testing Data in Chile", Volume 41 of Revista Mexicana de Astronomia y Astrofisica Conference Series, pp. 87-90 (2011).

[6] Bérthermin M., Daddi, E.., Magdis, M., et al., "A Unified Empirical Model for Infrared Galaxy Counts Based on the Observed Physical Evolution of Distant Galaxies" ApJ 757, L23 (2012).

[7] Parshley S.C., Adams, J., Nikola, T., Stacey, G.J., "The Opto-Cryo-Mechanical Design of the Short Wavelength Camera for the CCAT Observatory", Proc. SPIE, 9153 (2014).

[8] Wheeler, J., Koopman, B., Gallardo, P., et al., "Antireflection coatings for submillimeter silicon lenses", Proc. SPIE, 9153, (2014).

[9] Koopman, B., Gallardo, P., Cortes-Medellin, G., et al., "Deep reactive ion etching of silicon anti-reflection coatings fro sub-millimeter optics", Proc. SPIE, 9153 (2014).

[10] McKenney, C.M., Leduc, H.G., Dowell, C.D., et al., "The Current Status of MAKO”, Proc. SPIE, 9153, (2014).

[11] Rajagopalan, G., Kovacs, A., Monroe, R.M., Yoshida, H., Schoenwald, J., Nikola, T., Bradford, C.M., Golwala, S.R., Stacey, G.J., Zmuidzinas, J., "Readout electronics for the CCAT observatory's instruments at first light and beyond", Proc. SPIE, 9153, (2014).

[12] Kovacs, A,. Zmuidzinas, J., McKenney, C.M., Swenson, L.J., Hollister, M.I., Monroe, R.M., Dowell, C.D., Bradford, C.M., "Chirp readout for kinetic inductance detectors", Proc. SPIE, 9153 (2014). 